\title{
La logique de la pratique: les 15 ans de la Fondation EQUAM
}

\section{Marianne Jossen}

M.A., collaboratrice scientifique, Fondation EQUAM

\section{En 2014, EQUAM a fêté son $15^{\mathrm{e}}$ anniversaire. La Fondation promeut et certifie la} qualité dans le domaine de la médecine ambulatoire. Pour cet anniversaire, point de grande fête, mais une réflexion sur les clefs du succès, aujourd'hui et demain, en matière de travail sur la qualité. Eléments de réponse par un auditeur de la Fondation et, évidemment, des praticiens.

Il faut reconnaître à la pratique sa propre logique. Voilà l'argumentation de Pierre Bourdieu qui avait étudié la culture berbère durant les années 1950 [1]. Définissant la pratique comme action située, Bourdieu la voyait dépasser toute systématique. Toute classification, règle et instruction serait employée de manière plus ou moins flexible et adaptée à son contexte. Justement, la pratique aurait sa propre façon de faire, sa propre logique dans le maniement des aspects systématiques d'une culture. Dans le

«Nous ne contrôlons pas. Nous regardons et observons."

domaine médical, et plus spécifiquement en médecine générale, des tensions similaires peuvent être observées. Ici, l'évidence scientifique. Là, les patients individuels. Directives thérapeutiques et standards organisationnels d'un côté; travail animé par les évènements singuliers de l'autre.

\section{Le cœur du travail sur la qualité}

Travailler activement sur et au sein de cette tension et confronter tour à tour la pratique et la systématique: tel est le cœur de tout effort visant la qualité. C'est en tout cas un résumé des expériences réalisées par la Fondation EQUAM. Hans-Peter Wyss, qui est mandaté depuis 12 ans en tant qu'auditeur par la Fondation, commente: «Le cabinet classique vit dans des configurations de travail spontanées. Notre idée selon laquelle la standardisation est synonyme de sécurité a d'abord surpris de tels cabinets classiques.» La surprise est un élément décisif. Le travail sur la qualité ne peut réussir que si, d'une part, la systéma- tique a le potentiel de surprendre. D'autre part, la pratique quotidienne doit être ouverte à des questions critiques. Un sondage auprès des patients peut alors devenir une "expérience terriblement belle», comme le dit Michael Deppeler, médecin de famille certifié EQUAM.

\section{Hier et aujourd'hui}

La Fondation permet depuis 15 ans à des généralistes et médecins spécialisés de vivre de telles expériences. $\mathrm{Au}$ début, il s'agissait surtout de montrer qu'il était possible d'atteindre un haut niveau de qualité dans des cabinets encadrés par des systèmes de soins intégrés, décriés alors comme fournissant des prestations médicales bon marché. On adaptait et développait des programmes de qualité pour le cabinet entier et son organisation, mais aussi pour la prise en charge de malades chroniques ou la gestion de la médication. Aujourd'hui, EQUAM est une référence pour des cabinets qui veulent utiliser le management de qualité en tant qu'outil de direction et de réflexion. Les visites des auditeurs s'avèrent alors être un évènement important pour le cabinet entier: «Nos collaboratrices étaient fières que le travail qu'elles accomplissent durant toute l'année soit apprécié», raconte Suzanne Aebi, gynécologue travaillant dans un cabinet double à Bâle. Entre-temps, environ 300 cabinets répartis dans toute la Suisse alémanique sont certifiés. Il peut s'agir d'un institut de médecine du travail comptant autour de 90 collaboratrices ou d'un petit cabinet au fin fond des Grisons, comme celui où Claudia Schertlin s'occupe seule de ses patients. Elle affirme: «Je vois la certification comme un honneur et comme un encouragement à poursuivre continuel- 


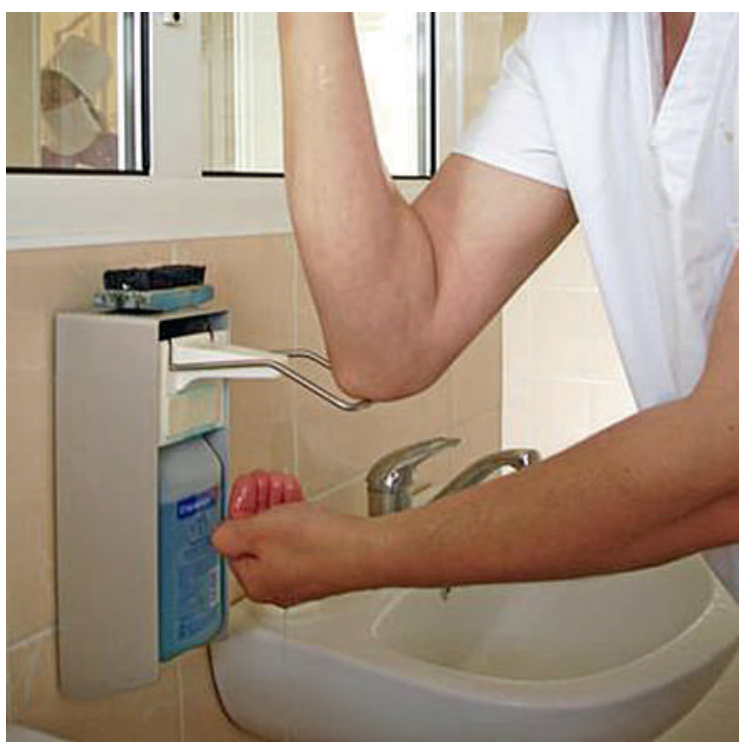

Le travail sur la qualité, c'est aussi observer et optimiser des processus et définir de nouvelles routines, par exemple dans le domaine de I'hygiène.

lement le développement de mon cabinet dans tous les domaines examinés.»

\section{Un peu de temps pour de nouveaux rituels}

Evidemment, la transparence qu'amène la procédure EQUAM a son prix, non seulement en termes monétaires mais surtout temporels. Ne peut-on pas continuer à travailler comme d'habitude? Faut-il tout réglementer? Non, il n'y a nullement lieu de tout

\section{Une gestion du personnel capitale au regard de la fluctuation et de la taille des cabinets.}

réglementer. Mais considérant la fluctuation grandissante du personnel et de la patientèle, il est judicieux de fonder certains processus sur des évidences médicales ou provenant des sciences de l'organisation. Fixer ces processus par écrit revient à les rendre indépendants des personnes. Et lorsqu'on intègre ces processus dans les routines quotidiennes, dans la "pratique» pour revenir à Bourdieu, ils deviennent des rituels. La différence importante aux pendants religieux et prémodernes se trouve dans le fait que ces rituels-là peuvent être remis en question et modifiés.

Dans le meilleur des cas, standard et pratique forment un engrenage. Hans-Peter Wyss cite l'hygiène en exemple. Les mesures d'hygiène sont des éléments du processus qui guide la patiente dans le cabinet. La définition des mesures structure le processus. Parallèlement, ces mesures protègent aussi bien les patients que les collaborateurs. On obtient ainsi en même temps «une sécurité des processus et une sécurité de l'hygiène».

\section{Le travail sur la qualité - un miroir de la pratique}

L'attitude des auditeurs est déterminante dans le processus traversé par le cabinet: «Nous ne contrôlons pas. Nous regardons et observons. C'est le personnel du cabinet qui décide de ce qu'il faut changer», précise Hans-Peter Wyss. Le travail sur la qualité est donc, d'une certaine manière, un miroir de la pratique, qui devrait rester entre les mains des praticiens. Cela demande un sens de l'initiative de la part des médecins et de l'assistanat médical. Michael Deppeler ajoute: "La définition de la qualité, sa mesure et son contrôle doivent se fonder sur l'approche médicale et le dialogue avec les patients.»

\section{La numérisation est un nouveau défi}

Le temps file et la Fondation compte aborder de manière aussi résolue les défis à venir. Depuis 2011 environ, la numérisation prend son essor au sein des cabinets, explique Hans-Peter Wyss. La gestion et la protection des données s'avèrent être de grands défis. La fluctuation du personnel et la taille des cabinets médicaux changent la donne en matière de ressources humaines. Comment, par exemple, gérer la communication lorsque la moitié du personnel se retrouve à travailler à temps partiel? Telles sont les questions que souhaite aborder la Fondation, en collaborant étroitement avec des praticiens en médecine ambulatoire, afin de développer de nouveaux programmes de qualité durant l'année à venir.

1 Voir Bourdieu P. Esquisse d'une théorie de la pratique. Précédée de trois études d'ethnologie kabyle. Editions du Seuil; 2000.

\section{Nécrologie}

Pendant la rédaction de cet article, nous avons appris le décès soudain, à l'âge de 72 ans, du Dr Kurt Hess, lic. oec., I'un des fondateurs d'EQUAM. Figure marquante de la Fondation, il était avant tout un ami pour beaucoup d'entre nous. Une nécrologie sera publiée dans l'un des prochains numéros du Bulletin des médecins suisses. 